\title{
CGRP-targeting antibodies reduce migraine frequency
}

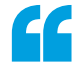 \\ CGRP pathway blockers \\ can be used \\ as tools to \\ understand \\ [migraine] and develop \\ further treatments}

Monoclonal antibodies (mAbs) that disrupt calcitonin gene-related peptide (CGRP) signalling can reduce the frequency of migraine attacks in patients with episodic or chronic migraine, according to two phase III trials that were recently published in The New England Journal of Medicine.

The neuropeptide CGRP is found in trigeminal nerve fibres surrounding intracranial blood vessels, where it functions as a vasodilator. CGRP is released from these nerve fibres during migraine attacks and has been shown to induce headaches in healthy individuals. Such observations have prompted a concerted effort to explore CGRP as a target for migraine therapy.

In the first of the two new trials, Stephen Silberstein and colleagues tested fremanezumab - a

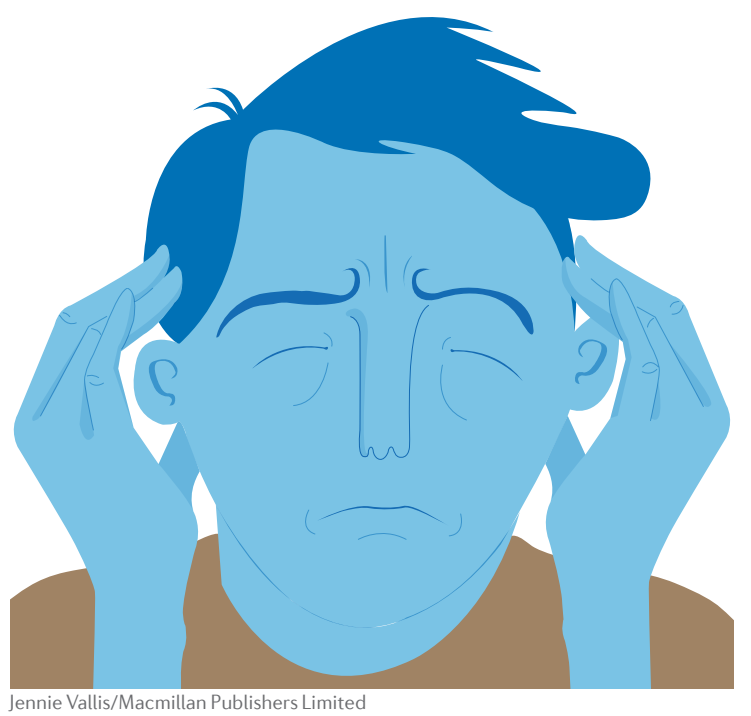

humanized $\mathrm{mAb}$ that targets CGRP - in 1,130 patients with chronic migraine. Following a 4-week baseline phase, the participants were randomly allocated to monthly or quarterly subcutaneous injections of the mAb ( $n=379$ and $n=376$, respectively) or to monthly injections of a placebo $(n=375)$ over a period of 12 weeks.

The primary end point of the trial was the change in the mean number of headache days per month between the baseline phase and the 12-week treatment period. Patients who received monthly injections of fremanezumab experienced the greatest mean reduction in monthly headache days (4.6-day reduction from a baseline of 13.2 days), followed by the group who received the quarterly dose (4.3 days, from a baseline of 12.8 days). By contrast, a mean reduction of only 2.5 headache days (from a baseline of 13.3 days) was reported in the placebo group.

"As in other trials of migraine treatment, eligibility was restricted to relatively healthy patients, so further studies will be needed to assess the safety and efficacy of fremanezumab in a population of patients with migraine and coexistent diseases," says Silberstein. "A study in patients with more-refractory disease is also being initiated."

In the second trial, a team led by Peter Goadsby tested erenumab - a human $\mathrm{mAb}$ that inhibits the CGRP receptor - in 955 patients with episodic migraine. The participants were randomly assigned to receive monthly injections of $70 \mathrm{mg}$ or $140 \mathrm{mg}$ erenumab $(n=317$ and $n=319$, respectively) or placebo $(n=319)$ for 6 months. The primary end point was the change in mean migraine days per month from baseline to months $4-6$ of the trial. The baseline values were 8.3 days for the two treatment groups and 8.2 days for the placebo group.

The reduction in migraine days was significantly greater in the erenumab-treated patients than in the placebo group (3.2 days for the $70 \mathrm{mg}$ dose and 3.7 days for the $140 \mathrm{mg}$ dose, compared with 1.8 days for placebo). In addition, erenumab treatment was associated with a decrease in the use of acute migraine-specific medication.

"This work shows that it is possible to design migraine-specific preventive agents rationally, based on a bench-to-bedside understanding of migraine biology," concludes Goadsby. "The implication is that CGRP pathway blockers can be used as tools to understand the disease and develop further treatments."

Heather Wood

ORIGINAL ARTICLES Silberstein, S. D. et al. Fremanezumab for the preventive treatment of chronic migraine. N. Engl.J. Med. 377, 2113-2122 (2017) |Goadsby, P. J. et al. A controlled trial of erenumab for episodic migraine. N. Engl.J. Med. 377, 2123-2132 (2017)

FURTHER READING Ho, T. W. et al. CGRP and its receptors provide new insights into migraine pathophysiology. Nat. Rev. Neurol. 6, 573-582 (2010) |Ashina, M. et al. Human models of migraine - short-term pain for long-term gain. Nat. Rev. Neurol. 13, 713-724 (2017) 